\title{
Optimization of plastic injection molding based on 3D reconstruction and finite element analysis
}

\author{
Sun Lingzhen, Ye Shuo* \\ ${ }^{1}$ Guangzhou Huali Science and Technology Vocational College, Guangzhou, China \\ ${ }^{2}$ Huali College Guangdong University of Technology, Guangzhou, China \\ Author for correspondence: Shuo Ye \\ Email:1603008553@qq.com
}

Keywords: Triangular mesh, Hole repair, Advancing Front Method, Triangulation, Mesh refinement

\begin{abstract}
In order to solve the problems on the holes caused by various factors in the 3D model, which have brought difficulties to the subsequent operation of the 3D model, a 3D hole repair method based on curvature feature is proposed. The basic concept of this algorithm is using Advancing Front Method to quickly fill holes for initial repair of the mesh, and then using the mesh optimization technology to adjust the initial mesh based on the curvature feature of the hole boundary point. First, according to the properties of the boundary edge in adjacent triangles, the boundary of the hole is identified, and then the Advancing Front Method and the relations between included angles of triangle top mark, the initial filling of the hole is completed. After that, combined with the curvature standard, refinement is made for hole mesh. Finally, geometry adjustment is made for the mesh top mark of repaired hole, to make it in natural transition with the surrounding mesh. Experiments show that the algorithm is simple and stable, as well as can complete the repair of different types of holes.
\end{abstract}

\section{Introduction}

The 3D mesh model is widely used to represent the 3D model because of its simple definition and strong description ability. Due to the problems in scanning and modeling, holes or other defects always exist in the mesh model, which brings difficulties to many subsequent operations such as model repair, finite element analysis and so on; therefore, the hole repair of mesh model is an important part of the early processing of 3D model. In recent years, scholars at home and abroad have done a lot of researches on the problem of 3D model hole repair, and obtained certain results [1-5]. Based on the analysis of these two kinds of methods, this paper presents a method of mesh model hole repair based on curvature feature. For this method, it firstly uses the definition of boundary edges in the mesh model to identify all the boundary points, and the boundary edges are connected to get the hole boundary. Then, the Advancing Front Method is adopted to make triangulation for hole area to get repair mesh, and then the curvature feature of the surrounding area is used to complete the refinement of repair mesh. Finally, the mesh's geometry is adjusted to better integrate repair mesh the surrounding area of the hole while maintaining the anisotropy.

\section{Identification of hole boundary}

Before repairing the hole, the boundary of the hole should be recognized first. Triangular mesh model is composed of a series of vertices and triangles connecting these vertices. The edge that just connects a triangle is called boundary edge, and the point on the boundary edge is known as the boundary point. Boundary triangle refers to the triangle that has one or two boundary points. According to the nature of the mesh model, after traversing the mesh surface, as shown in figure 1, the head and end of the boundary are connected to form the boundary of the hole. The specific implementation methods are as follows: 
Step1: for any vertex of the model surface, obtain the information of its adjacency point and adjacent triangle

Step2: compare the number of the adjacency point and adjacent triangles of this point. If it is equal, read the adjacency point to return step1; if not, mark $L$ as the boundary point, store the boundary point set and read the adjacency point, and repeat step 2

Step3: repeat the above steps until the set of boundary points does not produce any new point

Step4: connect the boundary edge, form a closed loop, and obtain the boundary of the hole

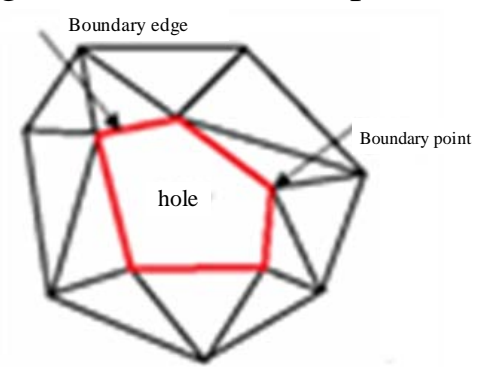

Fig. 1.Boundary point and boundary edge

\section{Triangulation of Advancing Front Method}

Triangulation is the key to solve the problem of hole repairing. For the traditional triangulation method, in order to get the optimized mesh in a one-time manner, various constraint conditions are added in the generated mesh model, for example, Liepa [4] uses the dynamic programming algorithm, and its high time complexity reaches $\mathrm{O}(\mathrm{n} 3)$. In this case, Advancing Front Method proposed by Lohner[11] and Lo[12] is carried out for the generation of initial mesh. With the recursive thinking, Advancing Front Method can effectively reduce the time complexity of problem, with advantages of simple implementation, fast speed, and good control. It has become one of the most important algorithms of mesh generation. The basic idea is to initialize the boundary of the triangulation region to be a set of opposite sides, called wavefront. With the current boundary as starting point, the triangle will be generated continuously, and before the wave is updated, the boundary will be narrowed until the wavefront is empty.

Advancing Front Method usually selects the vertex of the edge with the minimum angle as current point, and the implementation steps of the algorithm are as follows:

Step1: use the boundary point of the hole to initialize the wavefront

Step2: calculate included angle of each angle $v_{i}$ 's adjacent edges $\left(1, l_{i n}\right)$ of the wavefront

Step3: start with the vertices corresponding to the minimum angle, and generate triangles in the plane determined by $l_{i}$ and $l_{i+1}$ according to the rules in figure 2

Step4: add the wavefront unit of the newly constructed triangle to the wavefront unit collection

Step5: repeat step2 until the front unit is empty
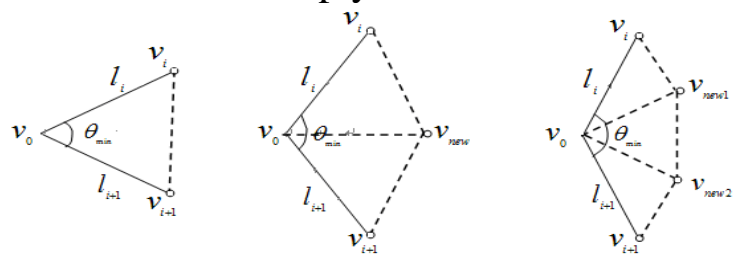

Fig. 2 Rules of newly added triangles

Advancing Front Method is based on the original hole boundary for triangulation, so the mesh generated by it can realize complete cover for the holes of any shape, so as to ensure the robustness of the algorithm. In the algorithm, $v_{i} v_{i+1}$ is directed edge newly generated according to the principle of minimum angle. $v_{\text {new }}, \quad v_{\text {new }}$ and $v_{\text {new } 2}$ are newly generated vertices, forming directed edge with the original vertices, and then joining the wavefront unit collection, to replace the original wavefront. Then, new frontwave is divided again until the hole polygon is divided into triangles. The maximum time complexity of this recursive algorithm is $\mathrm{O}(\mathrm{n})$, and the average time complexity 
is $\mathrm{O}(\operatorname{logn})$. Compared with the time complexity of the traditional optimization triangulation method $\mathrm{O}(\mathrm{n} 3)$, the speed of the hole triangulation is greatly improved.

However, in the process of repair, the minimum angle principle applied will result in uneven distribution of the generated meshes, which will affect the final repair results. Therefore, it is necesary to optimize the generated initial mesh.

\section{Conclusion}

This paper presents an efficient and robust 3D model hole filling algorithm. First, the definition of the boundary edge is used to identify the hole boundary, then the advancing front method is used to complete the initial repair of the hole. And according to the curvature standard, the initial mesh refinement is completed. Finally, according to the nature of the Delaunay, the mesh is optimized to get high-quality repaired mesh. The experimental results show that this method achieves the ideal repair results for different types of holes. In the next step, we will conduct in-depth research on the maintenance of the mesh feature and the continuous transition with the surrounding mesh, so as to obtain more ideal repair results.

\section{References}

[1] Yingyue Zhang, Qi Li, William J. Welsh, Prabhas V. Moghe, and Kathryn E. Uhrich. Micellar and Structural Stability of Nanoscale Amphiphilic Polymers: Implications for Anti-atherosclerotic Bioactivity, Biomaterials, 2016.

[2] Stephygraph, L.R., Arunkumar, N., Venkatraman, V. Wireless mobile robot control through human machine interface using brain signals. 2015 International Conference on Smart Technologies and Management for Computing, Communication, Controls, Energy and Materials, ICSTM 2015 Proceedings, art, 2015.

[3] Arunkumar, N., Balaji, V.S., Ramesh, S., Natarajan, S., Likhita, V.R., Sundari, S. Automatic detection of epileptic seizures using independent component analysis algorithm. IEEE-International Conference on Advances in Engineering, Science and Management, ICAESM-2012, 2012.

[4] Yang Du, Yizheng Chen, Yiyang Zhuang, Chen Zhu, Fujian Tang, *Jie Huang. Probing Nanostrain via a Mechanically Designed Optical Fiber Interferometer. IEEE Photonics Technology Letters, 2017.

[5] Dai Q. A tentative discussion on the cause of casing damage during the shale gas well cementing, Drilling Production Technology, 2015. 